\title{
MicroRNA-320c inhibits tumorous behaviors of bladder cancer by targeting Cyclin-dependent kinase 6
}

Xiao Wang, Jian Wu, Yiwei Lin, Yi Zhu, Xianglai Xu, Xin Xu, Zhen Liang, Shiqi Li, Zhenghui Hu, Xiangyi Zheng and Liping Xie*

\begin{abstract}
Background: Increasing evidence has suggested that dysregulation of microRNAs (miRNAs) could contribute to human disease including cancer. Previous miRNA microarray analysis illustrated that miR-320c is down-regulated in various cancers. However, the roles of miR-320c in human bladder cancer have not been well elucidated. Therefore, this study was performed to investigate the biological functions and molecular mechanisms of miR-320c in human bladder cancer cell lines, discussing whether it could be a therapeutic biomarker of bladder cancer in the future.

Methods: Two human bladder cancer cell lines and samples from thirteen patients with bladder cancer were analyzed for the expression of miR-320c by quantitative RT-PCR. Over-expression of miR-320c was established by transfecting mimics into T24 and UM-UC-3. Cell proliferation and cell cycle were assessed by cell viability assay, flow cytometry and colony formation assay. Cell motility ability was evaluated by transwell assay. The target gene of miR-320c was determined by luciferase assay, quantitative RT-PCR and western blot. The regulation of cell cycle and mobility by miR-320c was analyzed by western blot.
\end{abstract}

Results: We observed that miR-320c was down-regulated in human bladder cancer tissues and bladder cancer cell lines T24 and UM-UC-3. Over-expression of miR-320c could induce G1 phase arrest in UM-UC-3 and T24 cells, and subsequently inhibited cell growth. We also indentified miR-320c could impair UM-UC-3 and T24 cell motility. In addition, we identified CDK6, a cell cycle regulator, as a novel target of miR-320c. Moreover, we demonstrated miR-320c could induce bladder cancer cell cycle arrest and mobility via regulating CDK6. We also observed that inhibition of miR-320c or restoration of CDK6 in miR-320c-over-expressed bladder cancer cells partly reversed the suppressive effects of miR-320c.

Conclusions: miR-320c could inhibit the proliferation, migration and invasion of bladder cancer cells via regulating CDK6. Our study revealed that miR-320c could be a therapeutic biomarker of bladder cancer in the future.

Keywords: miR-320c, CDK6, Bladder cancer, Proliferation, Migration, Invasion

\section{Background}

Urinary bladder cancer is generally accepted as the 11th most commonly diagnosed type of cancer worldwide [1]. In the US, statistics illustrated that an estimated 74,690 cases were newly diagnosed bladder cancer, among which 15,580 were expected to die in 2014 [2]. Although it is believed that both environmental [3] and genetic factors

\footnotetext{
*Correspondence: xielp@zjuem.zju.edu.cn

Department of Urology, The First Affiliated Hospital, School of Medicine, Zhejiang University, 79 Qingchun Road, Hangzhou 310003, Zhejiang Province, People's Republic of China
}

(c) 2014 Wang et al.; licensee BioMed Central Ltd. This is an Open Access article distributed under the terms of the Creative Commons Attribution License (http://creativecommons.org/licenses/by/4.0), which permits unrestricted use, distribution, and reproduction in any medium, provided the original work is properly credited. The Creative Commons Public Domain Dedication waiver (http://creativecommons.org/publicdomain/zero/1.0/) applies to the data made available in this article, unless otherwise stated.
$[4,5]$, such as genetic polymorphism, chromosomal anomalies and epigenetic changes, play critical roles in the development of bladder cancer, the exact mechanisms of bladder carcinogenesis are still not well elucidated. Therefore, understanding the potential carcinogenetic mechanisms of these genetic changes is important to identify novel therapeutic targets and prognostic biomarkers.

MicroRNAs (miRNAs) are small (20 23 nucleotides), endogenous, non-coding RNAs, which constitute a novel cluster of target gene regulators [6]. They are involved in various cellular processes, including self-renewal, proliferation, 
metabolism and apoptosis, by inducing post-transcriptional gene repression via accelerating the degradation and/or blocking the translation of their target mRNAs [7]. The miRNA genes were observed to be specifically deleted in leukemia initially illustrated the important role of miRNA in carcinogenesis [8]. Subsequent researches have demonstrated that the expression of specific miRNAs is altered in many types of cancer, which is associated with carcinogenesis and cancer progression [9-13]. Meanwhile, accumulating evidences illustrated that the development and progression of bladder cancer is closely related to the aberrant expression of miRNAs [14]. The initial study of miRNA expression in bladder cancer was reported by Gottardo in 2007 and 10 up-regulated miRNAs were detected [15].

Previous miRNA microarray analysis illustrated that miR-320 is down-regulated in breast cancer, acute myelogenous leukemia and colon cancer, revealing that miR320 could probably act as a tumor suppressor in prohibiting the behavior of cancer [16-18]. It was reported that miR-320 could inhibit prostate cancer cell proliferation by down-regulating the Wnt/beta-catenin signaling pathway [19]. Additionally, miR-320a/c/d could inhibit the migration and invasion of hepatocellular cancer via targeting GNAI1, a crucial protein of multiple cellular signal transduction pathways [20]. Moreover, Iwagami et al. showed that miR-320c regulated the resistance of pancreatic cancer cells to gemcitabine via SMARCC1 (a core subunit of the switch/sucrose nonfermentable), suggesting that miR-320c could be a potential therapeutic target in pancreatic cancer [21]. Nevertheless, the potential mechanism of miR-320c in bladder cancer has not been well elucidated.

In our present study, we further testified miR-320c expression pattern in bladder cancer tissue. Additionally, for the first time, we detected that miR-320c could suppress growth and motility of the human bladder cancer cell line T24 and UM-UC-3. The tumor inhibitive role and potential mechanisms of miR-320c on bladder cancer were determined.

\section{Methods}

\section{Reagents}

The miR-320c mimic (named as miR-320c) and the negative control duplex (named as NC) lacking any significant homology to all known human sequences were used for transient gain of function research. For colony formation assay, the 2'-O-Methyl modified duplexes of both miR-320c and NC were used. 2'-O-Methyl modified miR-320c inhibitor (named as miR-320c-Inh) and $\mathrm{NC}$ inhibitor (named as Inh-NC) were used for observing the reversed effect of over-expression of miR-320c. The small interference RNA targeting human CDK6 mRNA (named as siCDK6) was synthesized as described previously [22], which targeted nucleotides 1424-1442 according to Genbank accession NM_001145306.1. All RNA duplexes were chemically synthesized by GenePharma Corporation (Shanghai, China). All the applied sequences were listed in Table 1.

\section{Tissue samples}

Paired bladder cancer tissues and para-carcinoma bladder mucosal tissues were acquired from patients receiving radical cystectomy. The samples were gained between Jan 2011 and June 2011 from the First Affiliated Hospital, School of Medicine, Zhejiang University (Hangzhou, P.R. China) with informed consent and Ethics Committee's approval. The clinical data of the patients were listed in Table 2. All tissue samples were stored in liquid nitrogen before use.

\section{Cell culture and transfection}

The human bladder cancer cell lines UM-UC-3, T24, and non-tumor urothelial cell line SV-HUC-1 (Shanghai Institute of Cell Biology, Chinese Academy of Sciences) were cultured in RPMI1640 medium (Gibco) containing $10 \%$ heat-inactivated fetal bovine serum (Gibco), 50U/ml penicillin and $50 \mu \mathrm{g} / \mathrm{ml}$ streptomycin under a humid atmosphere including $5 \% \mathrm{CO} 2$ at $37^{\circ} \mathrm{C}$. Cells were plated to $60-70 \%$ confluency in medium without antibiotics 1 day before transfection. Lipofectamine 2000 Reagent (Invitrogen, Carlsbad, CA, USA) was selected for transfection under the guide of the instruction.

Table 1 The oligonucleotides used in this study

\begin{tabular}{|c|c|}
\hline $\mathrm{Name}^{\mathrm{a}}$ & Sequence $\left(5^{\prime}->3^{\prime}\right)$ \\
\hline miR-320c mimics (sense) & AAAAGCUGGGUUGAGAGGGU \\
\hline NC (sense) & ACUACUGAGUGACAGUAGA \\
\hline miR-320c inhibitor & ACCCUCUCAACCCAGCUUUU \\
\hline microRNA inhibitor NC & CAGUACUUUUGUGUAGUACAA \\
\hline siCDK6 (sense) & CUGGAAAGGUGCAAAGAAAdTdT \\
\hline miR-320c F & AAAAGCTGGGTTGAGAGGGT \\
\hline U6 F & TGCGGGTGCTCGCTTCGGCAGC \\
\hline CDK6 F & GGATAAAGTTCCAGAGCCTGGAG \\
\hline CDK6 R & GCGATGCACTACTCGGTGTGAA \\
\hline GAPDH F & AAGGTGAAGGTCGGAGTCA \\
\hline GAPDH R & GGAAGATGGTGATGGGATTT \\
\hline CDK6-Wt F & $\begin{array}{l}\text { CAATCAATGCAAGAGTGATTGCAGCTTTA } \\
\text { TGTTCATTTMTTGTTGTIg }\end{array}$ \\
\hline CDK6-Wt R & $\begin{array}{l}\text { tcgacAACAAACAAACAAATGAACATAAA } \\
\text { GCTGCAATCACTCTTGCATTGATIgagct }\end{array}$ \\
\hline CDK6-Mut F & $\begin{array}{l}\text { CAATCAATGCAAGAGTGATTGgtcgaaat } \\
\text { TGTTCATTGTITTITGTg }\end{array}$ \\
\hline CDK6-Mut R & $\begin{array}{l}\text { tcgacAACAAACAAACAAATGAACAatttcgac } \\
\text { CAATCACTCTTGCATTGATTgagct }\end{array}$ \\
\hline
\end{tabular}

${ }^{\mathrm{a}} \mathrm{F}$, forward primer; $\mathrm{R}$, reverse primer. 
Table 2 Clinical data of the patients

\begin{tabular}{lllll}
\hline Patient no. & Sex & Age & TNM stage & Histological grade \\
\hline 1 & M & 62 & T2NOMO & II \\
2 & M & 60 & T1NOMO & I \\
3 & M & 53 & T1NOMO & II \\
4 & M & 86 & T1NOMO & II \\
5 & M & 55 & T1NOMO & ॥ \\
6 & F & 74 & T2NOMO & II \\
7 & M & 56 & T2NOMO & II \\
8 & F & 76 & T3NOMO & II \\
9 & M & 65 & T2NOMO & $\|$ \\
10 & F & 69 & T2NOMO & $\|$ \\
11 & M & 72 & T3NOMO & II \\
12 & M & 78 & T1NOMO & $\|$ \\
13 & M & 76 & T3NOMO & II \\
\hline
\end{tabular}

\section{RNA isolation and real-time PCR}

Expression level of miR-320c and CDK6 in tissues and cell lines was calculated by quantitative real-time RT-PCR. Small RNA was extracted from both frozen samples and cell lines with RNAiso Kit for Small RNA (TaKaRa, Japan) and subsequently reverse transcribed into cDNA with One Step PrimeScript miRNA cDNA Synthesis Kit (TaKaRa, Japan). Meanwhile, total RNA from cell lines UM-UC-3, T24, and SV-HUC-1 was extracted using RNAiso plus (TaKaRa, Japan) and transcribed into cDNA using PrimeScript RT reagent Kit (TaKaRa, Japan). The resulting cDNA of miR-320c and CDK6 was quantified by SYBR Premix Ex Taq (TaKaRa, Japan) via an ABI 7500 fast realtime PCR System (Applied Biosystems, Carlsbad, USA). Moreover, the cycle threshold $(\mathrm{Ct})$ value was used for our analysis $(\triangle \mathrm{Ct})$, and we determined the expression of small nuclear RNA U6 and GAPDH mRNA as internal controls to calculate the relative expression levels of miR-320c and CDK6 via the $2^{-\Delta \Delta C t}$ (delta-delta-Ct algorithm) method. All the primers were listed in Table 1.

\section{Cell viability assay}

Each well of 96-well plate was plated with 4000 cells (UM-UC-3 or T24). After $24 \mathrm{~h}$ incubation, the cells were transfected with RNA duplexes (25-100nM). After $48 \mathrm{~h}$ incubation, medium in each well was removed before cell counting solution (WST-8, Dojindo Laboratories, Tokyo, Japan) was added to it and incubated for another $2 \mathrm{~h}$. The absorbance of the solution was measured spectrophotometrically at $450 \mathrm{~nm}$ with MRX II absorbance reader (Dynex Technologies, Chantilly, VA, USA).

\section{Colony formation assay}

UM-UC-3 and T24 cells were incubated for $24 \mathrm{~h}$ after transfected with 2'-O-Methyl modified duplexes (50nM).
Five hundreds of transfected cells were seeded in a new six-well plate and cultivated continuously for another 10 days. Cells were subsequently treated with methanol and $0.1 \%$ crystal violet for fixing and staining. The colony formation rate was calculated via the following equation: colony formation rate $=$ (number of colonies $/$ number of seeded cells) $\times 100 \%$.

\section{Cell migration and invasion assay}

The 24-well Boyden chamber with $8 \mu \mathrm{m}$ pore size polycarbonate membrane (Corning, NY) was used for evaluating the cell motility. Matrigel was used to pre-coat the membrane to simulate a matrix barrier for invasion assay. Four thousands of cells were seeded on the upper chamber with $200 \mu \mathrm{l}$ serum-free medium after transfected with RNA duplex for 48 h. $600 \mu \mathrm{l}$ medium with $20 \%$ serum, served as a chemoattractant, was added to the lower chamber. After $24 \mathrm{~h}$ incubation, the membranes were fixed with methanol and stained with $0.1 \%$ crystal violet. Five visual fields $(\times 200)$ were randomly selected from each membrane, and the cell numbers were counted via a light microscope.

\section{Cell cycle analysis by flow cytometry}

After $48 \mathrm{~h}$ transfection, UM-UC-3 and T24 cells were washed with PBS and fixed in $75 \%$ ethanol at $-20^{\circ} \mathrm{C}$. After $24 \mathrm{~h}$ fixation, the cells were washed with PBS and treated with DNA Prep Stain (Beckman Coulter, Fullerton, CA) for $30 \mathrm{~min}$. Cell cycle analysis was conducted by BD LSRII Flow Cytometry System with FACSDiva software (BD Bioscience, Franklin Lakes, USA). The cell cycle distribution was illustrated as the percentage of cells in G1, S, and G2 populations and data was evaluated by ModFit LT software package.

\section{Protein extraction and Western blotting analysis}

After $48 \mathrm{~h}$ transfection with RNA duplexes, UM-UC-3 and T24 cells were lysed in cell lysis buffer and concentration of total protein in every lysate was quantified using the BCA Protein Assay kit (Pierce). Equivalent amounts $(30-50 \mu \mathrm{g})$ of protein were separated by $10 \%$ SDS-polyacrylamide gels and transferred to polyvinylidene difluoride membranes. Membranes were blocked for $1 \mathrm{~h}$ with $5 \%$ non-fat milk and then incubated at $4{ }^{\circ} \mathrm{C}$ overnight with specific primary antibody at appropriate dilutions according to the instructions. After washed three times in TBS-Tween, the membranes were incubated with the corresponding horseradish peroxidase (HRP)-conjugated secondary antibody for $1 \mathrm{~h}$ and detected by an enhanced chemi-luminescence (ECL) system (Pierce Biotechnology Inc., Rockford, IL). The primary immunoblotting antibodies used were: anti-GAPDH, antiCDK6 (Epitomics, Burlingame, CA). 


\section{Luciferase assays}

In order to construct the luciferase reporter vectors, the 3 ' UTR (untranslated region) of CDK6 was designed (Sangon, Shanghai, China), which contained putative target region for miR-320c (sequence set in Table 1). The synthesized oligonucleotide pair was annealed at $90^{\circ} \mathrm{C}$ for $3 \mathrm{~min}$ and then transferred to $37^{\circ} \mathrm{C}$ for another 15 min to form a duplex before inserted into pmirGLO Dual-Luciferase miRNA Target Expression Vector (Promega, USA) between the SacI and SalI sites. Additionally, the mutant miR-320c putative target region was also designed, annealed and inserted into pmirGLO Dual-Luciferase Vector in the same way (sequence set in Table 1). Both insertions were verified by sequencing (Sangon, Shanghai, China).

HEK 293 T cells were cultivated in a 24-well plate for $24 \mathrm{~h}$ before co-transfected with $50 \mathrm{nM}$ of either miR-320c mimic or NC oligos and $200 \mathrm{ng}$ reporter plasmid containing wild type (Wt) or mutant type (Mut) of CDK6 $3^{\prime}$-UTR. After $48 \mathrm{~h}$ transfection, the relative luciferase activity was calculated by Dual-Luciferase Reporter Assay System (Promega, USA).

\section{miR-320c inhibitor experiments}

To further verify the function of miR-320c, the antisense inhibitor (miR-320c inhibitor) experiments were performed to see whether the reverse effects to over-expression could be observed. The cells were co-transfected with either miR-320c mimics or NC oligos with miR-320c inhibitor or $\mathrm{NC}$ inhibitor [23]. After $48 \mathrm{~h}$ of transfection, colony formation assay, flow cytometry and transwell assay (cell migration and invasion assay) was used to analyze the cell proliferation, cell cycle and cell motility. Besides, expression level of miR-320c and CDK6 was calculated by quantitative real-time RT-PCR. In addition, the CDK6 expression was further determined by Western blotting.

\section{CDK6 rescue experiments}

The pTarget-CDK6 plasmid was constructed via inserting the human CDK6 coding sequence without the 3'-UTR into the pTarget vector (GeneCopoeia, USA), and verified by sequencing. The T24 cells were co-transfected with either miR-320c mimics or NC oligos with pTarget-CDK6 (pCDK6) or empty pTarget vector (pNull). After $48 \mathrm{~h}$ of transfection, colony formation assay, flow cytometry and transwell assay was used to evaluate the cell proliferation, cell cycle and cell motility. Additionally, the CDK6 expression was determined by Western blotting.

\section{Statistical analysis}

All the statistics were expressed as mean \pm standard deviation (SD) of three independent experiments. GraphPad Prism version 5 for Windows was used to conduct all the relative analyses via either the student's $t$-test or Two-way ANOVA. P $<0.05$ was considered to be statistically significant.

\section{Results}

\section{miR-320c is down-regulated in bladder cancer}

The expression pattern of miR-320c in human bladder cancer has not been analyzed. Therefore, we used realtime RT-PCR to quantify the expression levels of miR$320 \mathrm{c}$ in 13 pairs of human bladder cancer tissues and
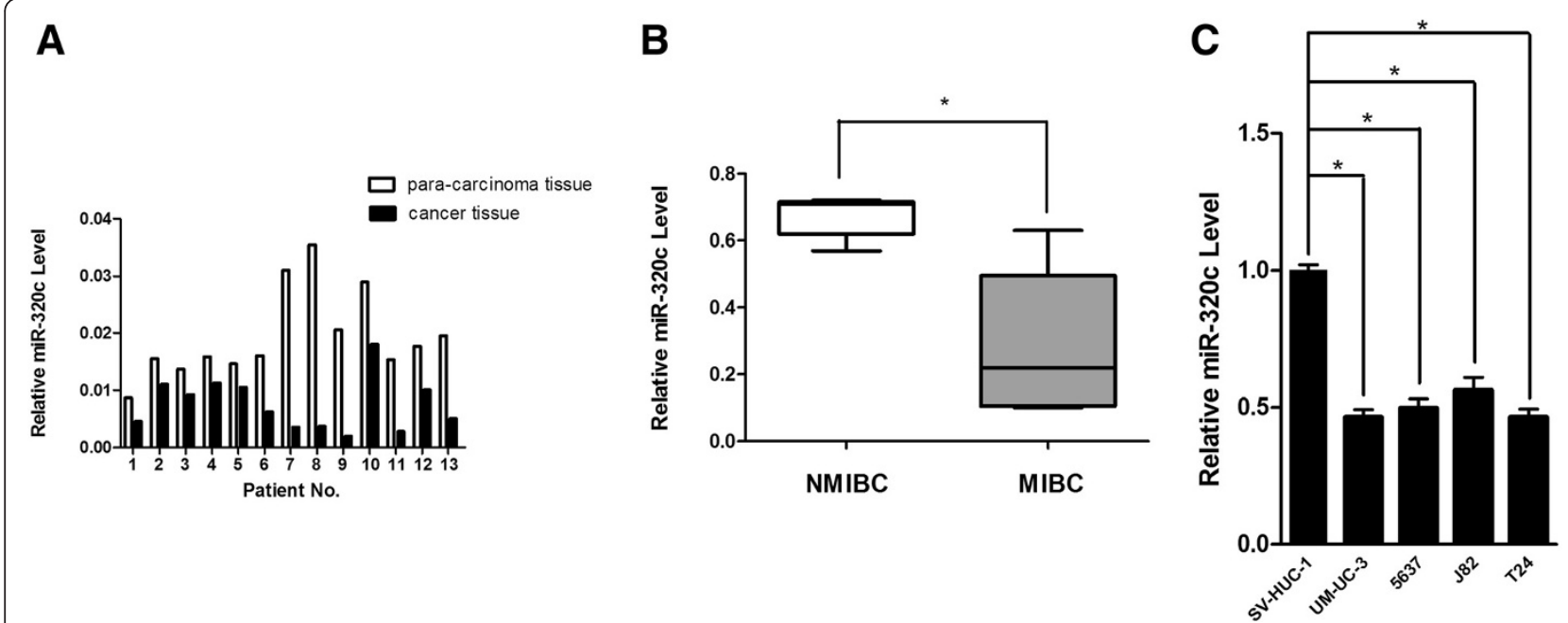

Figure $1 \mathrm{miR}-320 \mathrm{c}$ is down-regulated in bladder cancer Expression levels for miR-320c by real-time PCR analysis were normalized with U6. (A) Individual expression value of miR-320c for both cancer and matched normal tissues (calculated by $2^{-\Delta C t}$ ). (B) The relationship between NMIBC and MIBC was shown in a box and whiskers graph. Box-plot lines represented medians and interquartile ranges of the normalized threshold values, and whiskers indicated 10-90th percentiles. The expression level of miR-320c was significantly lower in MIBC compared with NMIBC. (C) The miR-320c levels in 4 bladder cancer cell lines were lower compared with SV-HUC-1 cell line. 
Table 3 Expression value of $\mathrm{miR}-320 \mathrm{c}$ in cancer and matched normal tissues (normalized by U6 RNA)

\begin{tabular}{llll}
\hline & Cancer tissues $\left(\mathbf{2}^{-\Delta C t}\right)$ & Normal tissues $\left(\mathbf{2}^{-\Delta C t}\right)$ & Folds $\left(2^{-\Delta \Delta C t}\right)$ \\
\hline 1 & 0.004581387 & 0.008668512 & 0.53 \\
2 & 0.011048543 & 0.015517070 & 0.71 \\
3 & 0.009226505 & 0.013696964 & 0.67 \\
4 & 0.011280697 & 0.015843117 & 0.71 \\
5 & 0.010525262 & 0.014578640 & 0.72 \\
6 & 0.006258358 & 0.016064279 & 0.39 \\
7 & 0.003569654 & 0.031034140 & 0.12 \\
8 & 0.003721242 & 0.035402621 & 0.10 \\
9 & 0.002008035 & 0.020617311 & 0.10 \\
10 & 0.018073253 & 0.028955877 & 0.63 \\
11 & 0.002800694 & 0.015303442 & 0.18 \\
12 & 0.010096506 & 0.017701311 & 0.57 \\
13 & 0.005083367 & 0.019505165 & 0.26 \\
\hline
\end{tabular}

adjacent normal mucosal tissues. Compared with their non-cancerous counterparts, it was observed that miR$320 \mathrm{c}$ expression levels were lower in cancerous tissues, and 6 out of 13 samples illustrated a 50\% reduction (Figure 1A). We also illustrated the expression value for both cancer and matched normal tissues for miR-320c normalized to U6 RNA in Table 3. In addition, we compared the expression pattern of miR-320c between muscle invasive bladder cancer (MIBC) and non muscle invasive bladder cancer (NMIBC), and we found the expression of miR-320c was lower in MIBC compared to NMIBC, which indicated that low level of miR-320c could be associated with tumor aggressiveness and poor prognosis (Figure 1B). However, such relationship should be further verified in a larger sample set in the future. Furthermore, 4 bladder cancer cell lines (UM-UC-3, T24, 5637 , J82) demonstrated similar expression pattern of miR-320c compared with non-tumor urothelial cell line SV-HUC-1 (Figure 1C). Therefore, it was speculated that miR-320c could be a potential tumor suppressor in bladder cancer.

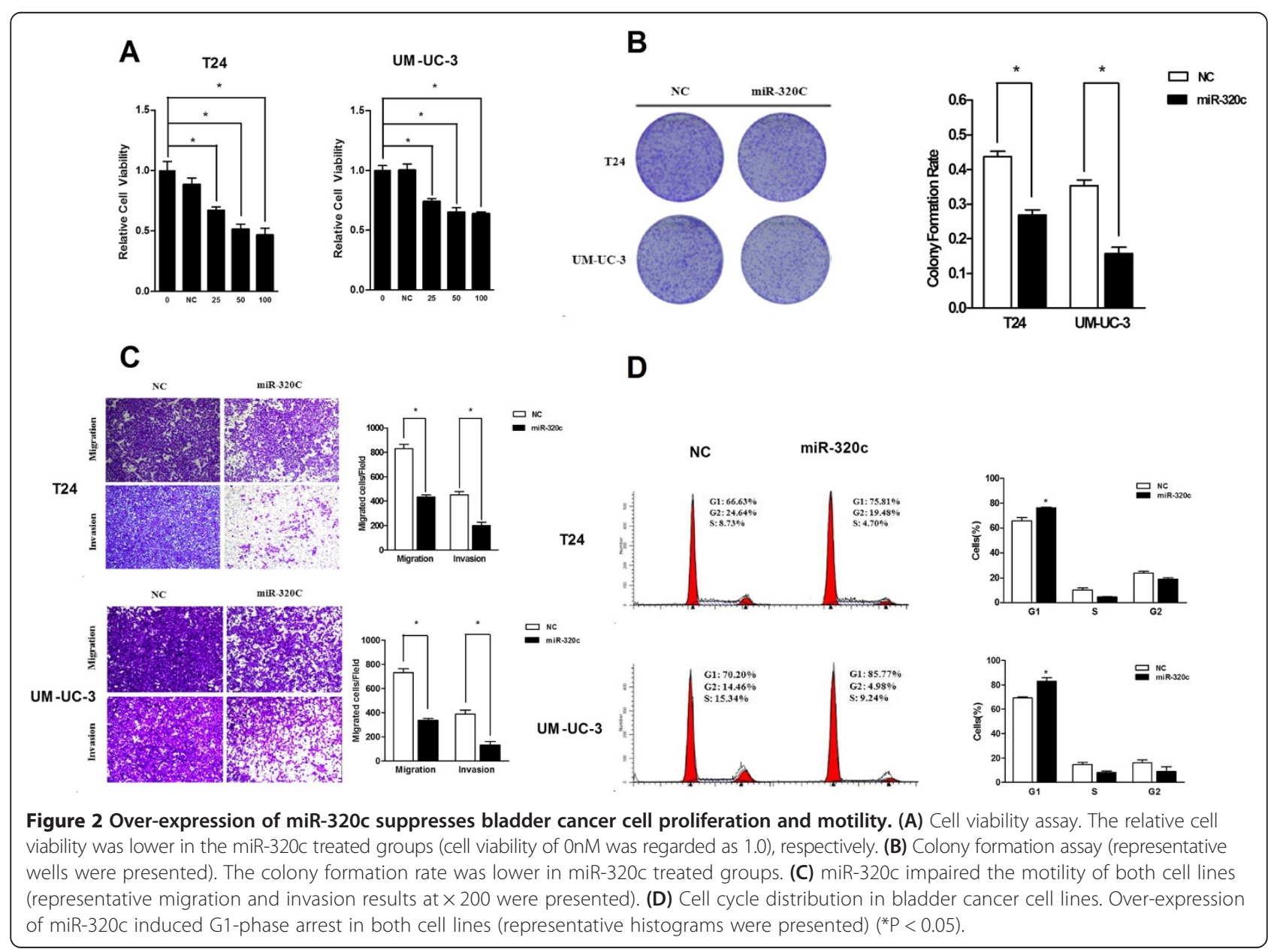


miR-320c suppresses bladder cancer cell viability, inhibits clone formation and triggers G1-phase arrest

In order to understand the potential mechanisms of miR-320c in tumor suppressing, the bladder cancer cell lines were transfected with miR-320c to evaluate the effect of over-expression via cell viability assay. As a result, miR-320c illustrated a significant inhibitory effect on bladder cancer cell viability in a dose-dependent manner (Figure 2A). After $48 \mathrm{~h}$ transfection, miR-320c (50nM) could reduce cell viability in both UM-UC-3 and T24 cell by $35 \%$ and $49 \%$, respectively. Furthermore, miR320c potently inhibited the colony forming ability in both cell lines. Compared with cell lines transfected with $\mathrm{NC}$, the colony formation rate decreased drastically in those transfected with miR-320c (Figure 2B).

Additionally, in order to better clarify the underlying mechanisms for miR-320c inhibiting cancer cell proliferation, we transfected the cells with 50nM miR-320c $48 \mathrm{~h}$ before assessing the impact of miR-320c on cell cycle distribution via flow cytometry. As a result, we observed a significant increase in the percentage of cells in the G1/G0 phase and a decrease in the percentage of cells in the $\mathrm{S}$ and $\mathrm{G} 2 / \mathrm{M}$ phase in miR-320c-overexpressing cells (Figure 2D). These results suggested that miR320 c could lead to G1-phase arrest.

\section{miR-320c impairs UM-UC-3 and T24 cell motility}

To further elucidate the function of miR-320c, we investigated the potential effect of miR-320c on UM-UC-3 and T24 cell motility. As illustrated by the transwell assay, over-expression of miR-320c decreased the migration and invasion of cancer cells compared with NC (Figure 2C). Therefore, miR-320c negatively regulated the motility of UM-UC-3 and T24 cells.

\section{CDK6 is a key regulator in miR-320c mediated cell proliferation suppression, cell cycle arrest and cell motility impairment}

We used TargetScan analysis (http://www.targetscan.org), miRTarBase (http://mirtarbase.mbc.nctu.edu.tw) and MicroCosm Targets (http://www.ebi.ac.uk/enright-srv/microcosm/ htdocs/targets/v5/) to detect the potential downstream targets of miR-320c. Among all the candidate genes predicted by the online tools, CDK6, a potential downstream target of miR-320c, was of particular interest

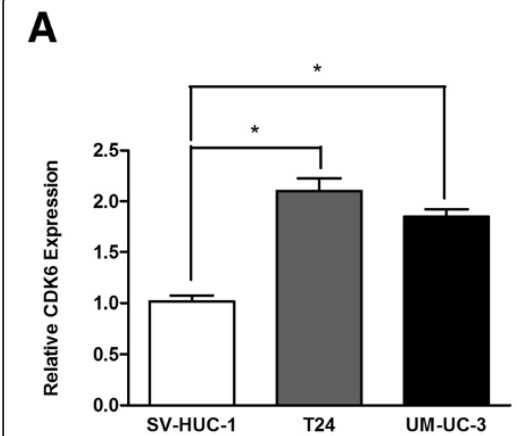

D
B

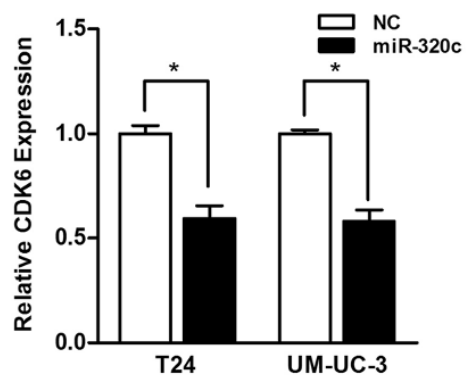

C

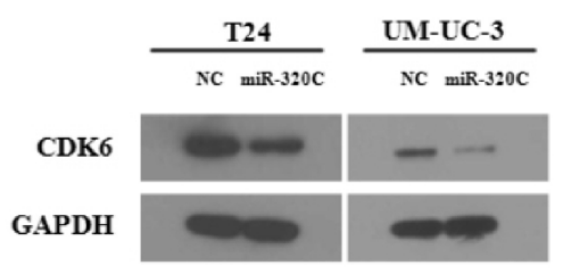

5' ...ATGCAAGAGTGATTGCAGCTTA... WT UTR IIIIII

3' TGGGAGAGTTGGGTCGAAAA miR-320c $D$

$55^{\prime}$...ATGCAAGAGTGATTGGTCGAAAA... Mut UTR

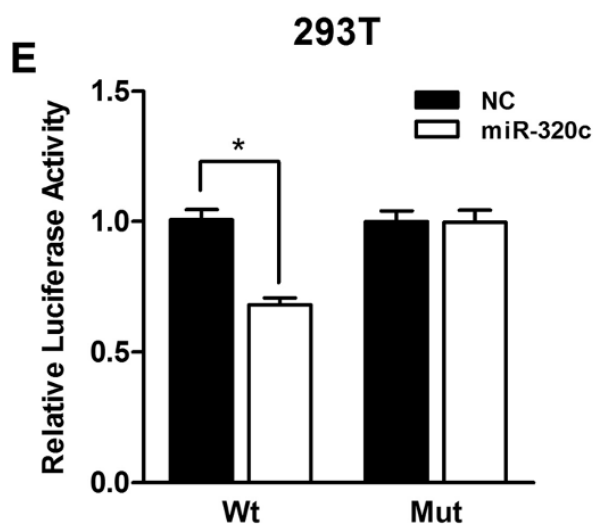

Figure 3 CDK6 is a direct target of miR-320c. (A) An increased expression pattern of CDK6 was observed in UM-UC-3 and T24 cells compared with SV-HUC-1 cells. (B, C) Over-expression of miR-320c reduced CDK6 expression level in both cell lines significantly (levels of mRNA and protein). (D) A predicted seed region in the $3^{\prime}$-UTR of CDK6 was illustratred (top). The mutated sequence was highlighted in underline (bottom). (E) $293 \mathrm{~T}$ cells were co-transfected with 50nM of either miR-320c mimic or NC oligos and 200 ng plasmid containing Wt or Mut of CDK6 3'-UTR. The relative firefly luciferase activity normalized with Renilla luciferase was calculated $48 \mathrm{~h}$ after transfection $\left({ }^{*} \mathrm{P}<0.05\right)$. 
because all online tools indicated that it had a very high scoring predicted binding site and CDK6 was considered to be a positive cell cycle regulator (G1/S transition) in many types of cancer [24-26]. Additionally, we also searched for information on conservation of CDK6 among species. The NCBI database illustrates that CDK6 gene is conserved in many species, including chimpanzee, dog, cow, mouse, rat, zebra fish, fruit fly, mosquito and C.elegans (http://www. ncbi.nlm.nih.gov/homologene/963). Previous study indicated that the expression of CDK6 increased drastically in bladder cancerous tissues compared with their noncancerous counterparts and elevated CDK6 expression resulted in the development of bladder cancer [26]. In our study, an increased expression pattern of CDK6 was observed in the human bladder cancer cell lines UM-UC-3 and T24 compared with non-tumor urothelial cell line SV-HUC-1 (Figure 3A). Moreover, we verified that the expression of CDK6 drastically reduced in both levels of mRNA and protein after the transfection of miR-320c, which was consistent with the cell cycle arrest phenomenon (Figure 3B, C).

\section{CDK6 is a novel direct target of miR-320c}

In order to clarify whether CDK6 was a direct downstream target of miR-320c, the synthesized 3'-UTR of CDK 6 was cloned into down-stream of firefly luciferase of pmirGLO Dual-Luciferase miRNA Target Expression Vector. Additionally, we also constructed another vector with mutated putative binding sites (Figure 3D). The results illustrated that HEK $293 \mathrm{~T}$ cells transiently transfected with the Wt-3'- UTR-reporter and miR-320c exhibited drastically reduced relative luciferase activity compared with co-transfection of Wt and NC. However, co-transfection of Mut CDK6 3'-UTR and miR-320c or NC did not affect the relative luciferase activity (Figure 3E). Therefore, CDK6 was considered to be a direct downstream target of miR-320c based on the luciferase assays.

\section{Inhibition of miR-320c partially reverses the over- expression of miR-320c induced effects}

To better verify the function of miR-320c, the antisense inhibitor (miR-320c inhibitor) experiments were performed to see whether the reverse effects to over-expression could be observed. As a result, co-transfection of miR-320c-Inh was applied to attenuate the miR-320c expression promotion and the CDK6 expression inhibition by miR-320c in the level of mRNA and protein (Figure 4A-C). Furthermore, miR-320c-Inh could partially reverse the effect of miR-320c on cell proliferation inhibition and cell cycle arrest in the T24 and UM-UC-3 cell lines (Figure 5A,B). A significant decrease in the percentage of cells in the G1/G0 phase and an increase in the G2/M phase was observed,

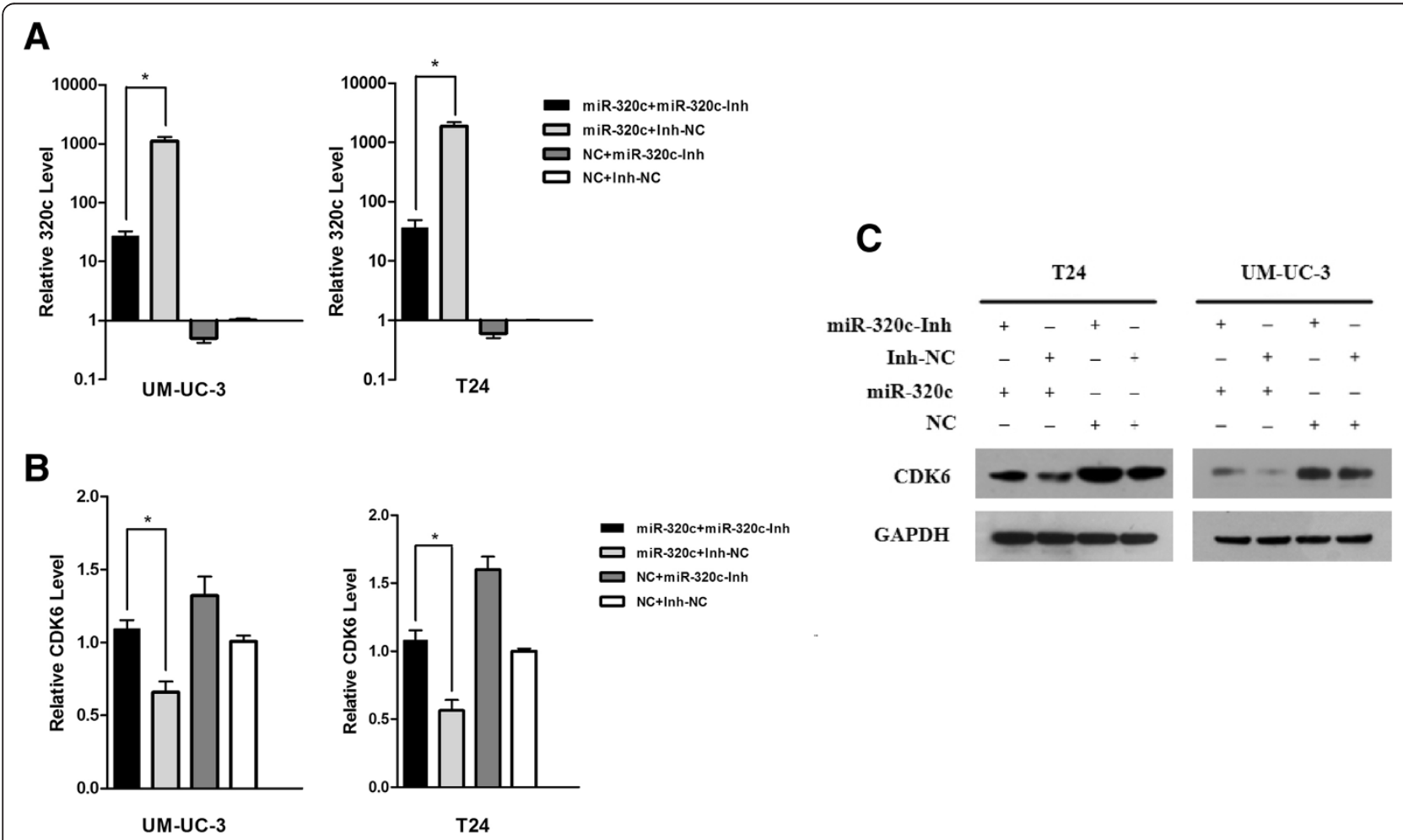

Figure 4 Ectopic miR-320c expression and inhibition of miR-320c suppress the expression of miR-320c and CDK6. T24 and UM-UC-3 cells were co-transfected with miR-320c-Inh (vs. Inh-NC) and miR-320c (vs. NC). (A) The expression of miR-320c was determined by real-time PCR. $(\mathbf{B}, \mathbf{C})$ The expression of CDK6 was determined by real-time PCR and western blot analysis. GAPDH served as an internal control $\left({ }^{*} \mathrm{P}<0.05\right)$. 


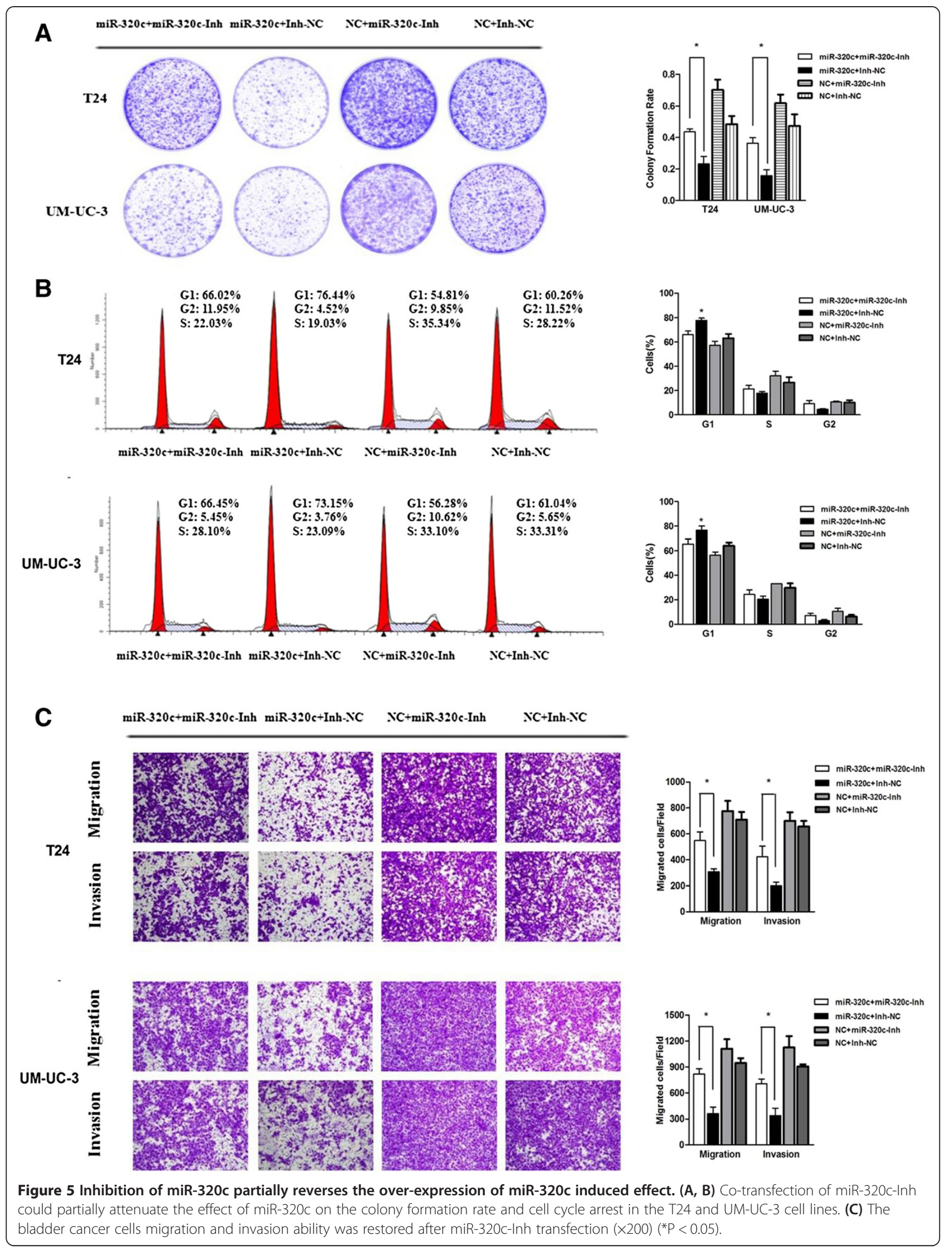


which indicating that transfection of miR-320c-Inh could attenuate the G1-phase arrest by miR-320c. Additionally, the bladder cancer cells migration and invasion ability was restored after miR-320c-Inh transfection (Figure 5C). Thus, we confirmed that miR-320c-Inh could reverse the effects to over-expression of miR-320c.

\section{Repression of CDK6 plays essential roles in miR-320c- induced bladder cancer inhibition effect}

Furthermore, we used loss of function approach to evaluate whether the physiological function of CDK6 was involved in miR-320c regulated cancer inhibition effect. The knock-down of CDK6 via RNAi technique dramatically decreased the expression of CDK6 in mRNA and protein levels in both cell lines (Figure 6A,B). Moreover, the transfection of siCDK6 significantly suppressed the proliferation of bladder cancer cell lines, and we also observed a significant increase in the percentage of cells in the G1/G0 phase and a decrease in the $S$ and G2/M phase, which phenocopied the effects of miR-320c on bladder cancer cells (Figure 6C-E). Interestingly, the knock-down of CDK6, generally accepted as a cell cycle mediator, also yield an inhibitory effect on cell invasion and migration (Figure 6F). Therefore, we further verified that miR-320c inhibited tumorous behaviors of bladder cancer cells by targeting CDK6.

\section{Restoration of CDK6 expression partially rescues} miR-320c-induced suppression of tumorous behavior We had verified that over-expression of miR-320c could induce G1-phase arrest, suppression of cell invasion and migration before and we wondered whether forced CDK6 expression could abrogate the cell cycle arrest and promote cell motility by miR-320c. In parallel, co-transfection of pCDK6 was applied to attenuate the CDK6 expression inhibition by miR-320c (Figure 7A). Forced CDK6 expression partially, but significantly, promoted cell proliferation and motility (Figure 7B, C). We also observed a significant decrease in the percentage of cells in the G1/G0 phase and an increase in the G2/M phase, which indicating that co-transfection of pCDK6 and miR-320c could attenuate the G1-phase arrest by miR-320c (Figure 7D). Thus, we confirmed that CDK6 was a key mediator of tumor suppression function of miR-320c in bladder cancer.

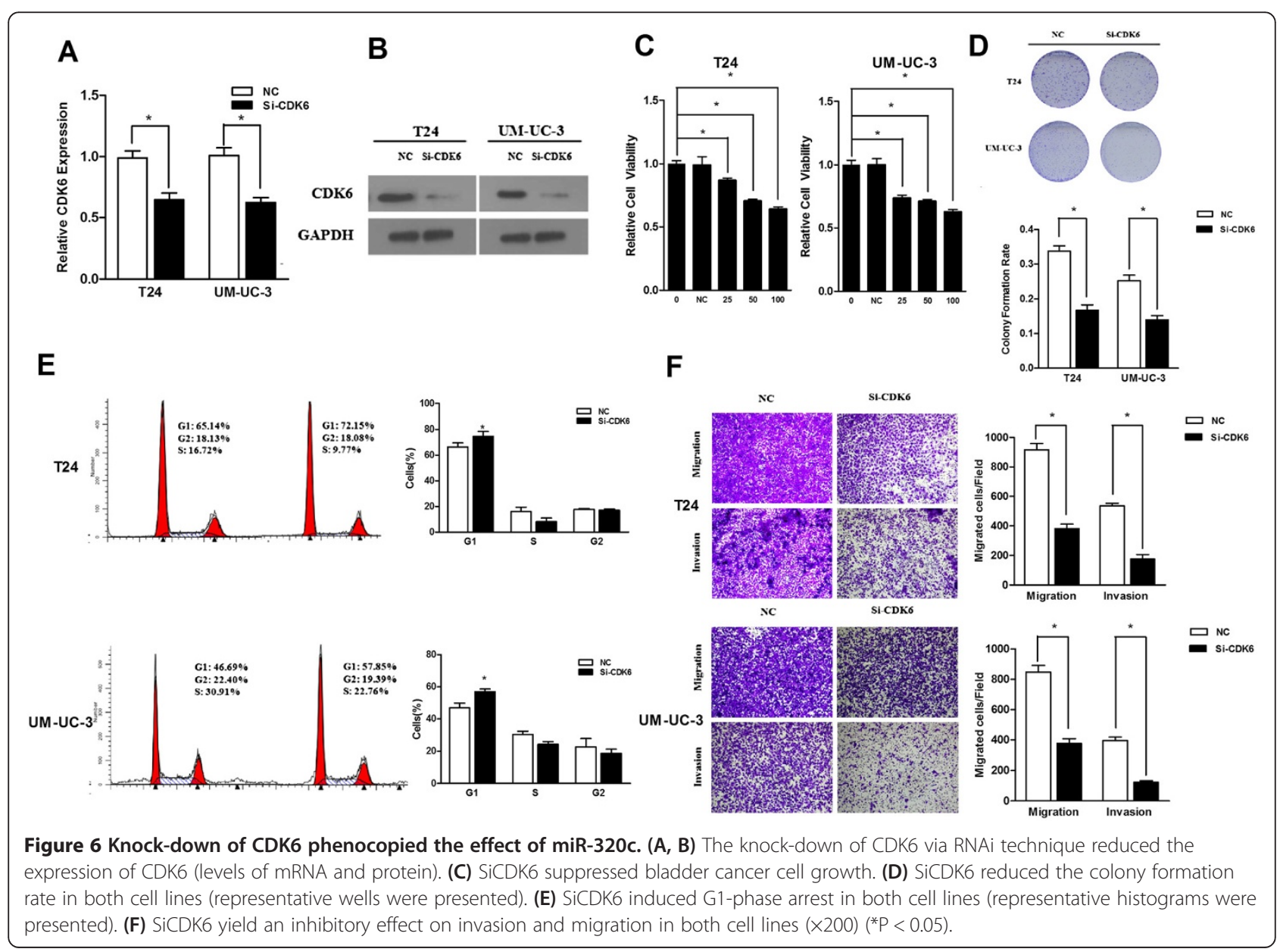




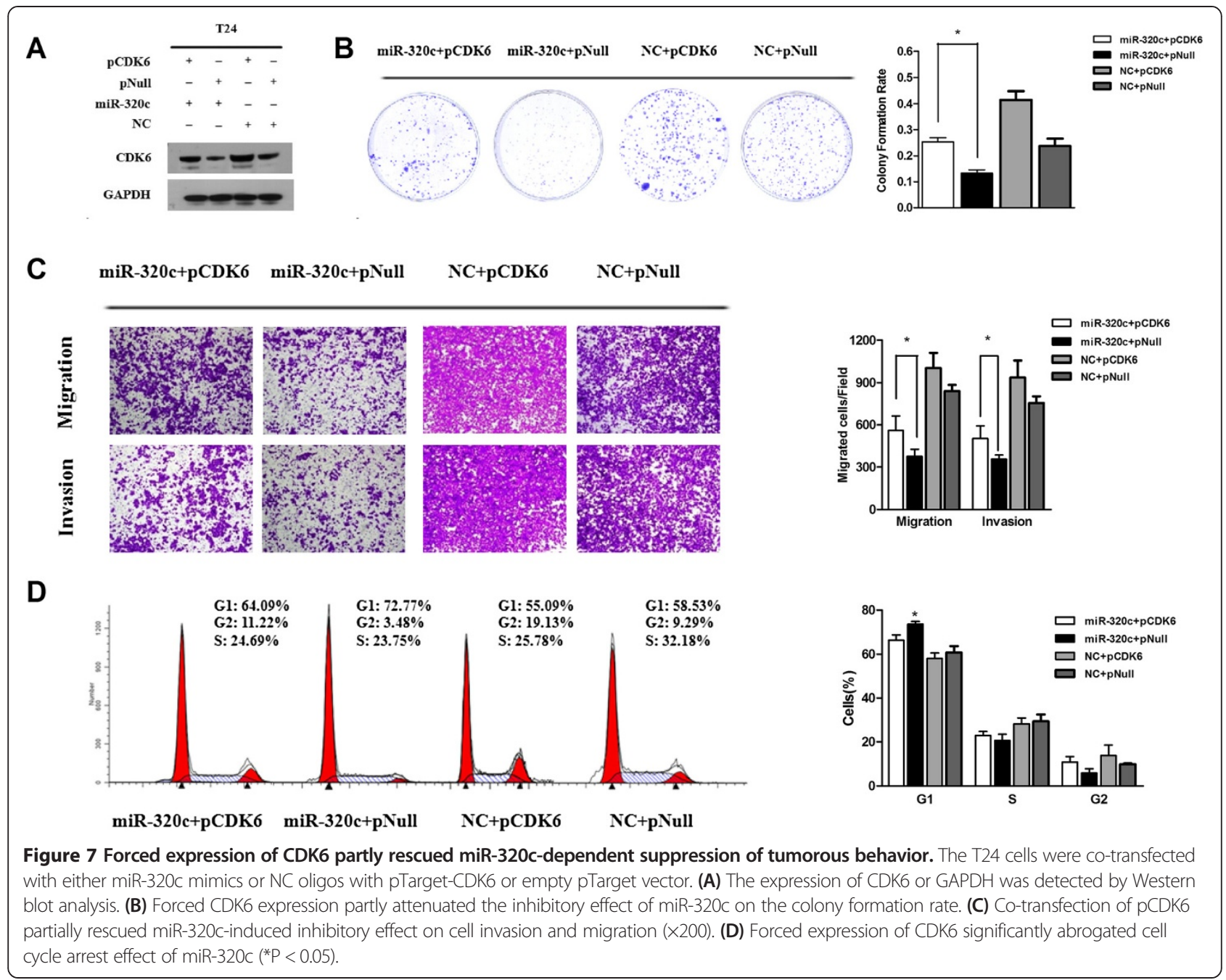

\section{Discussion}

During the past decades, effective targeted therapies of bladder cancer contributing to improved prognosis were the highlight of researches [27]. In recent years, a growing number of researches illustrated that abnormal expression of miRNAs was considered to be a key regulator in carcinogenesis $[28,29]$. Moreover, aberrant expression profiles of miRNA in cancer detected by microarray analysis provided deeper insights into the molecular passages of carcinogenesis $[17,18,30]$. A previous systematic review summarized the dysfunction of miRNAs in bladder cancer, which would help to establish a mature system in diagnosis and therapy using miRNAs in the future [14]. However, limited studies were focused on the regulative functional role of miRNAs in bladder cancer. The impact of specific miRNAs in bladder was still poorly understood. Thereafter, our institution performed some researches to elucidate the potential relationship between bladder cancer and miRNAs [31,32].

To the best of our knowledge, we initially detected a decreased expression pattern of miR-320c in human bladder cancer tissue compared with its normal adjacent tissue in the study, Recent miRNA microarray analyses demonstrated that miR-320 was down-regulated in many types of cancer, including breast cancer, acute myelogenous leukemia and colon cancer, indicating that miR320 could act as a tumor suppressor in cancer, which was similar to our results [16-18]. Furthermore, previous studies also revealed that miR-320c could inhibit the motility of hepatocellular cancer and regulate the resistance of pancreatic cancer cells to gemcitabine [20,21]. However, owing to unique genetic background in different types of cancer, the biological function of miR-320c in bladder cancer was not well elucidated. Therefore, this is the first study to determine the functional role of miR-320c in bladder cancer. Considering both of our tissue samples and cell lines are from patients with muscle-invasive bladder cancer, the outcome of this study is probably more meaningful in muscle-invasive or recurrent cancer.

Our study illustrated that miR-320c was down-regulated in bladder cancer tissues compared with normal adjacent 
tissues, though the sample size was relatively small. Similar result was detected in 4 bladder cancer cell lines compared with non-tumor urothelial cell line SV-HUC-1, which further strengthened the conclusion that miR-320c was down-regulated in bladder cancer. A gain-of- function study was further conducted in bladder cancer cell lines. When both UM-UC-3 and T24 cells were transfected with miR-320c, we observed that miR-320c could suppress bladder cancer cell viability and inhibit clone formation. In addition, flow cytometry indicated that miR-320c could trigger G1-phase arrest, which could be the potential mechanism of miR-320c-regulated proliferation inhibition. Moreover, cell motility assay demonstrated that overexpression of miR-320c impaired bladder cancer cells migration and invasion ability.

To elucidate the possible mechanism responsible for the anticancer behaviors triggered by miR-320c, we conducted a computerized analysis for the potential target. Therefore, we identified CDK6 as a new target of miR320. A previous study illustrated that CDK6 was overexpressed in bladder cancer tissue [26]. In our present study, similar expression pattern of CDK6 was observed in the human bladder cancer cell lines, which suggested the oncogenic role of CDK6 in bladder cancer. PCR and Western blot study indicated that miR-320c could dramatically inhibit CDK6 expression and luciferase assay further confirmed that CDK6 was a downstream target of miR-320c via directly binding to the 3 '-UTR.

To better verify the function of miR-320c, the antisense inhibitor (miR-320c inhibitor) experiments were performed. We confirmed that miR-320c-Inh could reverse the effects to over-expression of miR-320c. miR-320c-Inh could partially reverse the effect of miR-320c on cell cycle arrest and suppressing cell proliferation and motility.

As a critical cell cycle regulator, CDK6 induces an important cascade of events in G1-phase. It can modify $\mathrm{Rb}$ phosphorylation efficiently together with CDK4 and cyclin D1, and is considered to a primary sensor for driving cells through the $\mathrm{R}$ point to enter a new round of replication. Therefore, CDK6 has been regarded as a possible target for cancer therapy [33]. The knock-down of CDK6 via RNAi technique illustrated the G1-phase arrest, which phenocopied the cell cycle arrest effect of miR-320c overexpression.

Therefore, CDK6 is another important mediator in miR-320c induced G1/S phase transition arrest and cell proliferation suppression.

As we mentioned before, the knock-down of CDK6, generally accepted as a cell cycle mediator, also yielded an inhibitory effect on cell mobility, which was confusing. Previous studies also indicated that knock-down of CDK6 could inhibit cell invasion and migration in gastric and Ewing's Sarcoma [34]. However, the accurate mechanisms were still unknown. A recent study indicated that CDK6, as a key protein, coordinated cell proliferation and migration in breast cancer mainly dependent on the expression of estrogen receptor [35]. Furthermore, various oncogenic signaling pathways, including c-Myc, Ras, and Neu (ErbB2), have been described to converge on cell cycle proteins cyclinD1, CDK4/6 expression [36]. The data presented in our study also identified a novel role for cell cycle protein CDK6 in bladder cancer through the coordination of cell cycle, migration and invasion.

Ectopic over-expression of CDK6 (without the 3'UTR) significantly abrogated the miR-320c-induced G1 arrest of bladder cancer cells and promoted cell proliferation and motility in vitro. To sum up, these results suggested that miR-320c inhibited the proliferation and motility of bladder cancer cells via, at least in part, directly targeting the 3'-UTR of CDK6. Thus, our current study revealed what we believed to be a novel upstream regulatory mechanism of CDK6 in cancer cells.

\section{Conclusions}

In conclusion, our study suggests that miR-320c is a potential tumor suppressor in bladder cancer. By targeting CDK6, miR-320c can inhibit proliferation and impair cell mobility in bladder cancer cells. Restoration of miR-320c could be a promising therapeutic strategy for bladder cancer therapy.

\section{Abbreviations}

miRNA: MicroRNA; miR-320c: MicroRNA-320c; CDK6: Cyclin-dependent kinase 6; qPCR: Quantitative RT-PCR assays; WT: Wild type; Mut: Mutant type.

\section{Competing interests}

All authors declare that they have no competing interests.

\section{Authors' contributions}

$X W, Y W L, Z L$ and SQL performed and participated in analysis of laboratory experiments data. XW, JW and LPX participated in the design of experiments. $X W, X X L, X X$ and $Y Z$ acquired, preserved clinical samples. $Y W L, X Y Z$ and $L P X$ provided administrative support and funded experiments. XW, JW and $\mathrm{ZHH}$ drafted the manuscript. All authors have contributed and approved the final manuscript.

\section{Acknowledgements}

This study was supported by Grants from the National Key Clinical Specialty Construction Project of China, Combination of traditional Chinese and Western medicine key disciplines of Zhejiang Province (2012-XK-A23), Health sector scientific research special project (201002010), National Natural Science Foundation of China (Grant No. 81372773) and Natural Science Foundation of Zhejiang Province (LQ14H160012).

Received: 20 May 2014 Accepted: 18 August 2014

Published: 2 September 2014

\section{References}

1. Jemal A, Bray F, Center MM, Ferlay J, Ward E, Forman D: Global cancer statistics. CA Cancer J Clin 2011, 61(2):69-90.

2. Siegel R, Ma J, Zou Z, Jemal A: Cancer statistics, 2014. CA Cancer J Clin 2014, 64(1):9-29.

3. Wang $X$, Lin YW, Wang S, Wu J, Mao QQ, Zheng XY, Xie LP: A meta-analysis of tea consumption and the risk of bladder cancer. Urol Int 2013, 90(1):10-16

4. Chiong E, Kesavan A, Mahendran R, Chan YH, Sng JH, Lim YK, Kamaraj R, Tan TM, Esuvaranathan K: NRAMP1 and hGPX1 gene polymorphism and 
response to bacillus Calmette-Guerin therapy for bladder cancer. Eur Urol 2011, 59(3):430-437.

5. Casadio V, Molinari C, Calistri D, Tebaldi M, Gunelli R, Serra L, Falcini F, Zingaretti C, Silvestrini R, Amadori D, Zoli W: DNA Methylation profiles as predictors of recurrence in non muscle invasive bladder cancer: an MS-MLPA approach. J Exp Clin Cancer Res 2013, 32:94.

6. Bartel DP: MicroRNAs: genomics, biogenesis, mechanism, and function. Cell 2004, 116(2):281-297.

7. Bartel DP: MicroRNAs: target recognition and regulatory functions. Cell 2009, 136(2):215-233.

8. Calin GA, Liu CG, Sevignani C, Ferracin M, Felli N, Dumitru CD, Shimizu M, Cimmino A, Zupo S, Dono M, Dell'Aquila ML, Alder H, Rassenti L, Kipps TJ, Bullrich F, Negrini M, Croce CM: MicroRNA profiling reveals distinct signatures in B cell chronic lymphocytic leukemias. Proc Natl Acad Sci US A 2004, 101(32):11755-11760.

9. Zhou B, Chen H, Wei D, Kuang Y, Zhao X, Li G, Xie J, Chen P: A novel miR-219-SMC4-JAK2/Stat3 regulatory pathway in human hepatocellular carcinoma. J Exp Clin Cancer Res 2014, 33(1):55

10. Ma C, Nong K, Wu B, Dong B, Bai Y, Zhu H, Wang W, Huang X, Yuan Z, Ai K: miR-212 promotes pancreatic cancer cell growth and invasion by targeting the hedgehog signaling pathway receptor patched-1. J Exp Clin Cancer Res 2014, 33:54.

11. Wang Z, Wang J, Yang Y, Hao B, Wang R, Li Y, Wu Q: Loss of has-miR-337$3 p$ expression is associated with lymph node metastasis of human gastric cancer. J Exp Clin Cancer Res 2013, 32:76.

12. Li Y, Chao $Y$, Fang $Y$, Wang J, Wang $M$, Zhang $H$, Ying $M$, Zhu $X$, Wang $H$ : MTA1 promotes the invasion and migration of non-small cell lung cancer cells by downregulating miR-125b. J Exp Clin Cancer Res 2013, 32:33.

13. Gao SM, Yang JJ, Chen CQ, Chen JJ, Ye LP, Wang LY, Wu JB, Xing CY, Yu K: Pure curcumin decreases the expression of WT1 by upregulation of miR-15a and miR-16-1 in leukemic cells. J Exp Clin Cancer Res 2012, 31:27.

14. Catto JW, Alcaraz A, Bjartell AS, De Vere WR, Evans CP, Fussel S, Hamdy FC, Kallioniemi O, Mengual L, Schlomm T, Visakorpi T: MicroRNA in prostate, bladder, and kidney cancer: a systematic review. Eur Urol 2011, 59(5):671-681.

15. Gottardo F, Liu CG, Ferracin M, Calin GA, Fassan M, Bassi P, Sevignani C, Byrne D, Negrini M, Pagano F, Gomella LG, Croce CM, Baffa R: Micro-RNA profiling in kidney and bladder cancers. Urol Oncol 2007, 25(5):387-392.

16. Yan LX, Huang XF, Shao Q, Huang MY, Deng L, Wu QL, Zeng $Y X$, Shao JY: MicroRNA miR-21 overexpression in human breast cancer is associated with advanced clinical stage, lymph node metastasis and patient poor prognosis. RNA 2008, 14(11):2348-2360.

17. Schepeler T, Reinert JT, Ostenfeld MS, Christensen LL, Silahtaroglu AN, Dyrskjot L, Wiuf C, Sorensen FJ, Kruhoffer M, Laurberg S, Kauppinen S, Orntoft TF, Andersen CL: Diagnostic and prognostic microRNAs in stage II colon cancer. Cancer Res 2008, 68(15):6416-6424.

18. Schaar DG, Medina DJ, Moore DF, Strair RK, Ting Y: miR-320 targets transferrin receptor 1 (CD71) and inhibits cell proliferation. Exp Hematol 2009, 37(2):245-255.

19. Hsieh IS, Chang KC, Tsai YT, Ke JY, Lu PJ, Lee KH, Yeh SD, Hong TM, Chen $Y L$ : MicroRNA-320 suppresses the stem cell-like characteristics of prostate cancer cells by downregulating the Wnt/beta-catenin signaling pathway. Carcinogenesis 2013, 34(3):530-538.

20. Yao J, Liang LH, Zhang Y, Ding J, Tian Q, Li JJ, He XH: GNAl1 Suppresses Tumor Cell Migration and Invasion and is Post-Transcriptionally Regulated by Mir-320a/c/d in Hepatocellular Carcinoma. Cancer Biol Med 2012 9(4):234-241.

21. Iwagami $Y$, Eguchi $H$, Nagano $H$, Akita H, Hama N, Wada H, Kawamoto K, Kobayashi S, Tomokuni A, Tomimaru Y, Mori M, Doki Y: miR-320c regulates gemcitabine-resistance in pancreatic cancer via SMARCC1. Br J Cancer 2013, 109(2):502-511.

22. Zhu Y, Lu Y, Zhang Q, Liu JJ, Li TJ, Yang JR, Zeng C, Zhuang SM: MicroRNA$26 \mathrm{a} / \mathrm{b}$ and their host genes cooperate to inhibit the G1/S transition by activating the pRb protein. Nucleic Acids Res 2012, 40(10):4615-4625.

23. Chen X, Wang X, Ruan A, Han W, Zhao Y, Lu X, Xiao P, Shi H, Wang R, Chen L, Chen S, Du Q, Yang H, Zhang X: miR-141 is a key regulator of renal cell carcinoma proliferation and metastasis by controlling EphA2 expression. Clin Cancer Res 2014, 20(10):2617-2630.
24. Zhu X, Li Y, Shen H, Li H, Long L, Hui L, Xu W: miR-137 inhibits the proliferation of lung cancer cells by targeting Cdc42 and Cdk6. FEBS Lett 2013, 587(1):73-81.

25. Lapointe J, Lachance $Y$, Labrie $Y$, Labrie C: A p18 mutant defective in CDK6 binding in human breast cancer cells. Cancer Res 1996, 56(20):4586-4589.

26. Wang G, Zheng L, Yu Z, Liao G, Lu L, Xu R, Zhao Z, Chen G: Increased cyclin-dependent kinase 6 expression in bladder cancer. Oncol Lett 2012, 4(1):43-46.

27. Prasad SM, Decastro GJ, Steinberg GD: Urothelial carcinoma of the bladder: definition, treatment and future efforts. Nat Rev Urol 2011, 8(11):631-642

28. Koturbash I, Zemp FJ, Pogribny I, Kovalchuk O: Small molecules with big effects: the role of the microRNAome in cancer and carcinogenesis. Mutat Res 2011, 722(2):94-105.

29. Zhang M, Yang Q, Zhang L, Zhou S, Ye W, Yao Q, Li Z, Huang C, Wen Q Wang J: miR-302b is a potential molecular marker of esophageal squamous cell carcinoma and functions as a tumor suppressor by targeting ErbB4. J Exp Clin Cancer Res 2014, 33:10.

30. Yang N, Kaur S, Volinia S, Greshock J, Lassus H, Hasegawa K, Liang S, Leminen A, Deng S, Smith L, Johnstone CN, Chen XM, Liu CG, Huang Q, Katsaros D, Calin GA, Weber BL, Butzow R, Croce CM, Coukos G, Zhang L: MicroRNA microarray identifies Let-7i as a novel biomarker and therapeutic target in human epithelial ovarian cancer. Cancer Res 2008, 68(24):10307-10314.

31. Lin Y, Chen H, Hu Z, Mao Y, Xu X, Zhu Y, Wu J, Li S, Mao Q, Zheng X, Xie L: miR-26a inhibits proliferation and motility in bladder cancer by targeting HMGA1. FEBS Lett 2013, 587(15):2467-2473.

32. Li S, Xu X, Hu Z, Wu J, Zhu Y, Chen H, Mao Y, Lin Y, Luo J, Zheng X, Xie L: MicroRNA-490-5p inhibits proliferation of bladder cancer by targeting c-Fos. Biochem Biophys Res Commun 2013, 441(4):976-981.

33. Landis MW, Pawlyk BS, Li T, Sicinski P, Hinds PW: Cyclin D1-dependent kinase activity in murine development and mammary tumorigenesis. Cancer Cell 2006, 9(1):13-22.

34. Zhang Z, Huang L, Yu Z, Chen X, Yang D, Zhan P, Dai M, Huang S, Han Z, Cao K: Let-7a functions as a tumor suppressor in Ewing's sarcoma cell lines partly by targeting cyclin-dependent kinase 6. DNA Cell Biol 2014, 33(3):136-147.

35. Lamb R, Lehn S, Rogerson L, Clarke RB, Landberg G: Cell cycle regulators cyclin D1 and CDK4/6 have estrogen receptor-dependent divergent functions in breast cancer migration and stem cell-like activity. Cell Cycle 2013, 12(15):2384-2394.

36. Wang C, Lisanti MP, Liao DJ: Reviewing once more the c-myc and Ras collaboration: converging at the cyclin D1-CDK4 complex and challenging basic concepts of cancer biology. Cell Cycle 2011, 10(1):57-67.

\section{doi:10.1186/s13046-014-0069-6}

Cite this article as: Wang et al:: MicroRNA-320c inhibits tumorous behaviors of bladder cancer by targeting Cyclin-dependent kinase 6 Journal of Experimental \& Clinical Cancer Research 2014 33:69.

\section{Submit your next manuscript to BioMed Central and take full advantage of:}

- Convenient online submission

- Thorough peer review

- No space constraints or color figure charges

- Immediate publication on acceptance

- Inclusion in PubMed, CAS, Scopus and Google Scholar

- Research which is freely available for redistribution 